\section{Disección endoscópica submucosa para el tratamiento de neoplasias incipientes del tubo digestivo: experiencia en un centro universitario de alto volumen}

\author{
RICARDO MEJÍA ${ }^{1,2}$, JOSEFINA SÁEZ ${ }^{\mathrm{a}}$, \\ PAMELA BRIONES ${ }^{2}$, ENRIQUE NORERO ${ }^{2}$, MARCO CERONI $^{2}$, \\ ALFONSO DÍAZ², ALLAN SHARP ${ }^{1}$
}

\section{Experience with endoscopic submucosal dissection for the treatment of early neoplasms of the digestive tract}

Background: Endoscopic submucosal dissection (ESD) allows en-bloc resection of early gastro-intestinal neoplasms (EGIN) with healing potential. Aim: To describe the results of patients treated with ESD for EGIN by our team. Patients and Methods: Descriptive study of patients with EGIN who underwent ESD with curative intention between January 2008 and March 2020. Results: One hundred thirty-two ESD were performed in 127 patients. $77 \%$ were gastric lesions, $14 \%$ colorectal, $8 \%$ esophageal and 1\% duodenal. En-bloc resection was achieved in $98.4 \%$ of ESDs. Eighty eight percent of patients met curative standards. Overall, cancer-specific, and recurrence-free survival were 95\%, 100\% and 98\% respectively. Conclusions: ESD allows en-bloc resections with curative potential in selected patients, but with a significant reduction in morbidity and mortality and less impact on quality of life. Our results suggest the feasibility to perform ESD in our country with results comparable to those reported in the literature.

(Rev Med Chile 2021; 149: 501-507)

Key words: Endoscopy: Endoscopic Mucosal Resection; Gastrointestinal Neoplasms.

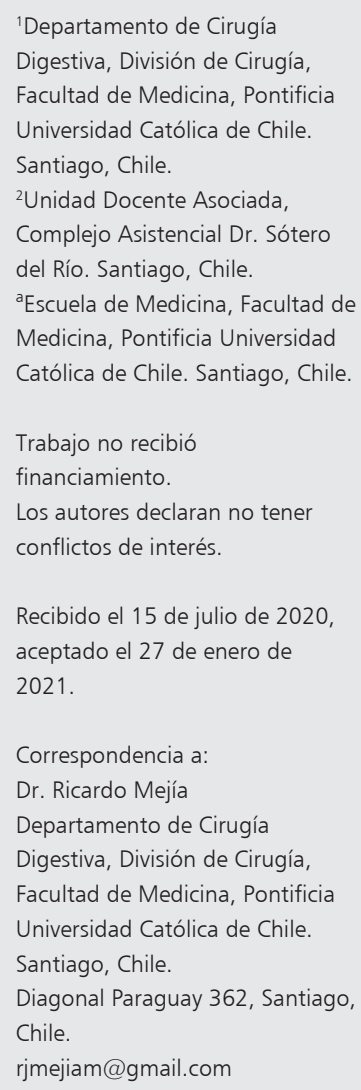

L as neoplasias malignas del tubo digestivo son responsables de $25 \%$ de la mortalidad anual por cáncer en Chile ${ }^{1}$. El pronóstico de este tipo de cánceres es pobre, debido a que la mayoría de los casos se presentan en etapas avanzadas ${ }^{2-4}$. Lograr el diagnóstico en estadios precoces, donde el tratamiento está asociado a mejores resultados oncológicos, es fundamental para disminuir la mortalidad asociada a estas neoplasias ${ }^{5,6}$. En este sentido, tanto en Chile como en el mundo, se han instituido programas de tamizaje para la detección precoz de tumores del tubo digestivo. Lo anterior, ha determinado un aumento en la pesquisa de lesiones preneoplásicas y neoplásicas incipientes, que pueden ser tratadas mediante la resección endoscópica, con riesgos significativamente menores para los pacientes y un pronóstico comparable a la cirugía tradicional ${ }^{7,8}$.

Un porcentaje importante de las neoplasias incipientes del tubo digestivo (NITD) corresponde a lesiones pequeñas, susceptibles de ser extirpadas a través de resección endoscópica mucosa (EMR, por sus siglas en inglés $)^{9-11}$. Esta técnica consiste en la remoción de un fragmento de mucosa y parte variable de la submucosa, mediante el uso de un lazo ${ }^{11}$. Si bien la EMR es factible en lesio- 
nes de mayor tamaño, se asocia a resecciones en fragmento ${ }^{10,11}$. Esto aumenta las tasas de recidiva e impide un adecuado análisis histológico ${ }^{9,10}$. La disección endoscópica submucosa (DES) es una técnica desarrollada en Japón que subsana este problema en cuanto permite la resección en bloque de lesiones incipientes del tubo digestivo ${ }^{12}$. La extracción en una sola pieza, que incluye la totalidad de la mucosa y la submucosa, se traduce en un mejor estudio anatomopatológico y en que al certificar una resección completa ésta podría tener potencial curativo ${ }^{12,13}$.

La mayoría de las series clínicas de DES provienen de Asia, donde la DES constituye el estándar de tratamiento para NITD $^{7,8,14,15}$. El análisis de las series reportadas muestra que la DES es una técnica segura con buenos resultados oncológicos en el largo plazo $^{8,12-15}$. En occidente, especialmente en Latinoamérica y Chile, la experiencia reportada es escasa ${ }^{16-20}$.

El propósito de este trabajo es describir los resultados perioperatorios y oncológicos de pacientes sometidos a DES por NITD por nuestro grupo.

\section{Material y Método}

La presente comunicación corresponde a un estudio observacional descriptivo de una serie de casos consecutivos de pacientes con NITD sometidos a DES. Los pacientes fueron tratados en el Complejo Asistencial Dr. Sótero del Río y Red de Salud UC-Christus en el período comprendido entre enero de 2008 y marzo de 2020. La indicación de resección endoscópica fue evaluada en un comité multidisciplinario, luego de una discusión individualizada y contando con una adecuada etapificación clínica. Todas las resecciones fueron realizadas por endoscopistas con experiencia en DES del tracto digestivo.

Se registraron datos epidemiológicos y perioperatorios, obtenidos desde el registro institucional.

\section{Técnica endoscópica}

Todos los pacientes fueron sometidos a la DES en pabellón quirúrgico, utilizando un panendoscopio Olympus ${ }^{\circledR}$ modelo GIF-H180 o GIF-HQ190 en el caso de las disecciones esofágicas, gástricas, duodenales y de colon descendente o recto. En el caso de lesiones de colon derecho y transverso, se utilizó un colonoscopio Olympus ${ }^{\circledR}$ modelo
CF-HQ190L. Luego de identificar la lesión y sus márgenes con endoscopía de luz blanca y cromoendoscopía (utilizando índigo carmín o lugol sin diluir), se procedió a la marcación de la periferia con electrocoagulación. Esta maniobra no fue utilizada rutinariamente en las lesiones colónicas. Luego de elevar la lesión mediante inyección de una solución constituida por Voluven ${ }^{\circledR}$, índigo carmín a $1,2 \%$ y adrenalina a $0,4 \%$ en el espacio submucoso, se efectuó mucotomía y posterior disección submucosa con cuchillos endoscópi$\cos$ (FlushKnife ${ }^{\circledR}$ BT de $2,5 \mathrm{~mm}$ o $2,0 \mathrm{~mm}$ y/o DualKnife-J ${ }^{\circledR}$ de 2,0 mm o 1,5 mm) procurando la resección en bloque de la pieza. Al terminar el procedimiento se realizó hemostasia del lecho con pinza de coagulación CoaGrasper ${ }^{\circledR}$ y se verificó la indemnidad de la pared (Figura 1).

La pieza fue extendida en una superficie de plumavit, fijada en sus extremos con alfileres y sumergida en formalina para su estudio anatomo-patológico definitivo (Figura 1). Las piezas fueron analizadas por varios patólogos del Servicio de Anatomía Patológica del Hospital Sótero del Río. Se utilizó tinción con hematoxilina-eosina y se evaluó dirigidamente el tipo histológico, tamaño tumoral, grado de diferenciación, presencia de permeaciones linfovasculares y los bordes laterales y profundo. Se consideraron R0 las resecciones que no presentaron compromiso neoplásico de los márgenes laterales ni profundos, independiente de la distancia de la lesión al borde.

Los pacientes fueron trasladados a una unidad quirúrgica de baja complejidad y mantenidos las primeras $24 \mathrm{~h}$ en régimen cero. Se evitó el uso de anticoagulantes y se favoreció la deambulación precoz. El día posterior a la cirugía los pacientes se realimentaron y el alta fue indicada regularmente a las $48 \mathrm{~h}$.

\section{Seguimiento}

El seguimiento fue realizado mediante control clínico y endoscópico de acuerdo a la ubicación e histología de la lesión resecada.

\section{Análisis estadístico}

Fueron analizadas variables demográficas, características clínicas, del diagnóstico endoscópico e histológico preoperatorio, datos del procedimiento y morbilidad asociada, resultado histológico definitivo y seguimiento. Se evaluó la sobrevida global y libre de enfermedad. 


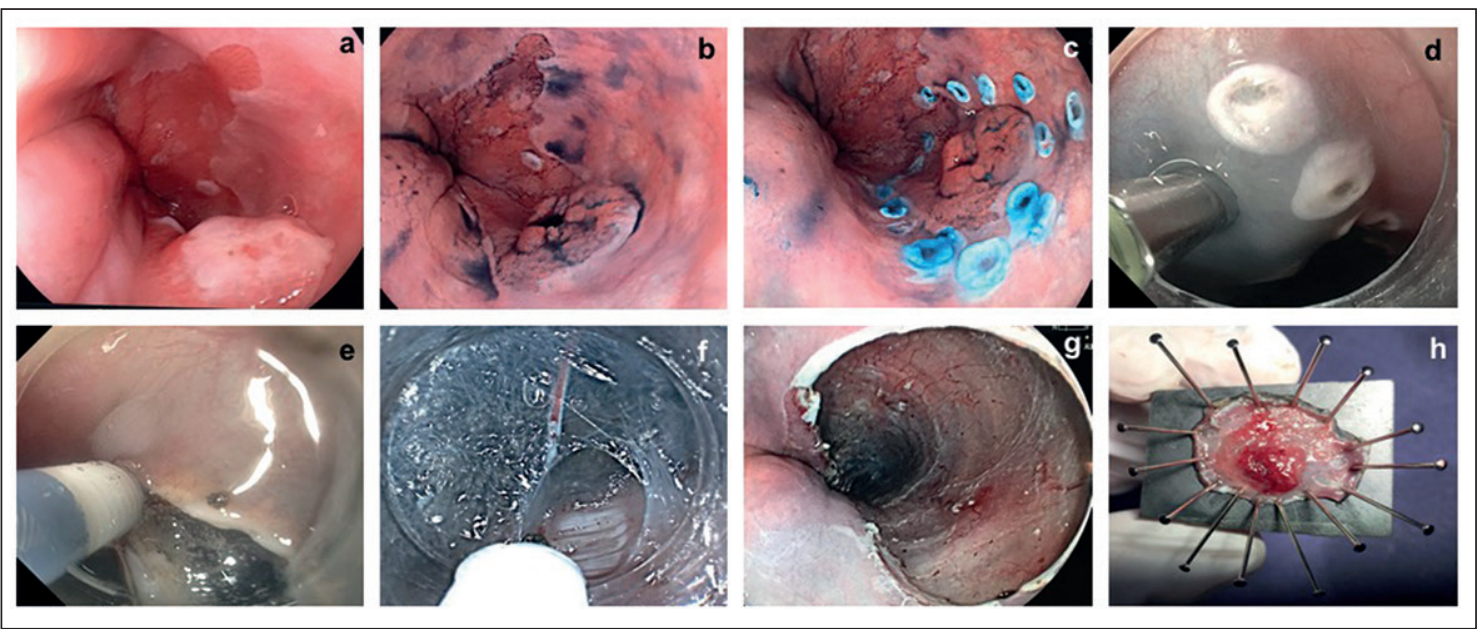

Figura 1. Técnica de Disección Endoscópica Submucosa. (a) Luz blanca. (b) Cromoendoscopía. (c) Marcación. (d) Elevación submucosa. (e) Mucotomía. (f) Disección submucosa. (g) Lecho. (h) Pieza extendida.

Las variables categóricas se expresan en valores numéricos y porcentajes, las variables continuas en medianas y rangos. El análisis estadístico se realizó con SPSS v24, Inc, Chicago, IL.

El presente trabajo respetó las normas éticas concordantes con la Declaración de Helsinki y fue aprobado por el Comité Ético Científico del Servicio de Salud Metropolitano Sur Oriente. Todos los pacientes firmaron un documento de consentimiento informado previo a la cirugía.

\section{Resultados}

Un total de 132 DES en 127 pacientes por NITD fueron realizadas entre los años 2008 y 2020. El 46\% de los pacientes fueron hombres y la edad promedio fue de 68 años (40-91). Respecto del riesgo quirúrgico/anestésico, considerando la clasificación de la American Society of Anesthesiologists (ASA), 74\% fueron clasificados como ASA II y $13 \%$ ASA III.

Del total de resecciones, $77 \%$ corresponde a DES gástricas, $14 \%$ a lesiones colorrectales, $8 \%$ esofágicas y $1 \%$ duodenales (Figura 2).

\section{Esófago}

Un total de 10 pacientes fueron sometidos a una DES por cáncer de esófago entre noviembre de 2010 y febrero de 2020. La histología pre-ope- ratoria incluyó 4 carcinomas escamosos, 4 adenocarcinomas y 2 lesiones con displasia de alto grado (DAG).

La resección en bloque fue lograda en todos los casos. No se presentó morbilidad ni mortalidad perioperatoria. Un paciente evolucionó con una estenosis esofágica, manejada favorablemente con dilataciones endoscópicas.

Todos los pacientes fueron dados de alta al segundo día postoperatorio.

Todas las resecciones fueron catalogadas

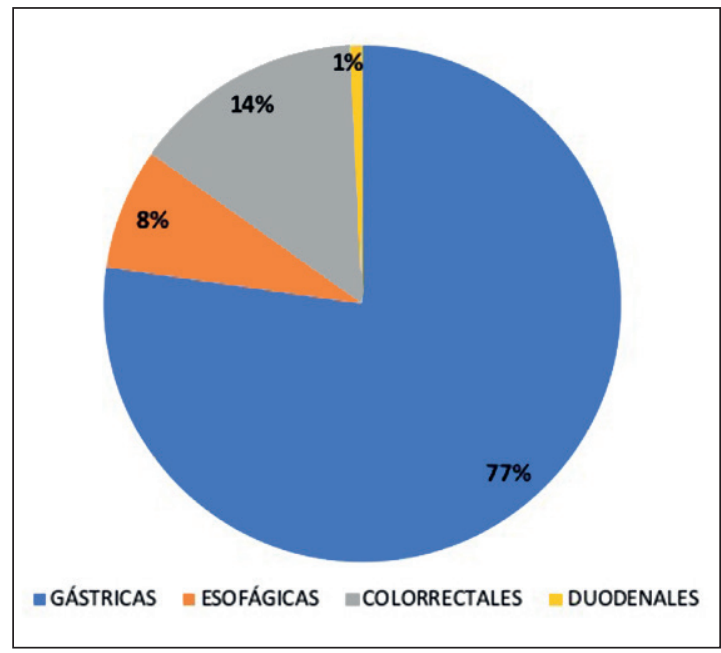

Figura 2. Porcentaje de lesiones según ubicación. 
como R0 y en $90 \%$ de los pacientes se consideró la DES como curativa de acuerdo a la anatomía patológica. En un solo caso se informó compromiso submucoso profundo (1.600 micras) y el paciente fue sometido a una esofagectomía como tratamiento definitivo. Su estudio histológico final informó ausencia de tumor esofágico residual y de adenopatías.

A la fecha no se han diagnosticado recurrencias. El promedio de seguimiento fue de 38 meses (3,5-123). La sobrevida global de la serie alcanzó $90 \%$, solo registrándose un caso de mortalidad secundario a un accidente cerebrovascular tres años después de la intervención. La sobrevida específica por cáncer esofágico fue de $100 \%$ y la sobrevida libre de recurrencia fue $100 \%$ (Tabla 1).

\section{Estómago}

Un total de 102 DES fueron realizadas en 98 pacientes entre enero de 2008 y marzo de 2020. La ubicación más frecuente correspondió al tercio medio con $37 \%$. El tercio superior e inferior representaron $30 \%$ y $35 \%$ respectivamente.

La histología pre-operatoria incluyó 68 adenocarcinomas, 30 lesiones con displasia de alto grado (DAG), 2 con displasia de bajo grado y 2 tumores neuroendocrinos.

La resección en bloque fue lograda en $98 \%$ de los casos. En 2 pacientes esto no fue alcanzado; en un caso por imposibilidad de elevar la submucosa y un segundo paciente presentó un shock anafiláctico durante la disección que obligó a suspender el procedimiento.

Cuatro pacientes (4\%) presentaron hemorragia digestiva manejada satisfactoriamente por vía endoscópica.

Durante los primeros años de desarrollo de la técnica, entre el 2008 y 2014, no se contaba con insuflador de $\mathrm{CO}_{2}$. En este período 4 pacientes presentaron perforaciones advertidas de la pared gástrica, debiendo ser manejados mediante gastrorrafia laparoscópica. Desde que el insuflador de $\mathrm{CO}_{2}$ estuvo disponible, este tipo de eventos pueden ser resueltos durante el mismo procedimiento con clips endoscópicos. La tasa de perforaciones que han requerido manejo quirúrgico ha descendido a $1 \%$.

Un paciente presentó una estenosis pilórica durante el seguimiento y presentó mala respuesta a dilataciones endoscópicas repetidas por lo que debió ser sometida a una gastro-yeyuno anasto- mosis laparoscópica. No se presentaron casos de mortalidad postoperatoria.

La mediana de estadía hospitalaria fue de 2 días, con un rango entre 1 y 40 días.

Del total de las resecciones en bloque, en siete pacientes la biopsia informó borde lateral o profundo positivo, por lo tanto $93,2 \%$ fue catalogada como R0. Uno de ellos se encuentra en seguimiento con biopsias subsecuentes negativas. Otro fue sometido a una nueva DES que resultó curativa. Los restantes cinco fueron sometidos a tratamiento quirúrgico, pero solo en uno se demostró en la histología definitiva la presencia de un adenocarcinoma intramucoso residual.

De acuerdo a los criterios expandidos ${ }^{13}, 85 \%$ de las disecciones submucosas cumplieron con estándares de curación. Siete pacientes fueron sometidos a gastrectomía con disección ganglionar, solo uno de ellos demostró compromiso ganglionar. En tres pacientes con resecciones R0 no curativas, se decidió seguimiento dada su edad avanzada y comorbilidades.

Tres pacientes de la serie presentaron recurrencias tardías (12, 26 y 51 meses). Uno de ellos se sometió a una segunda DES que resultó curativa y los otros dos fueron sometidos a gastrectomía, ambas biopsias definitivas resultaron sin neoplasia.

El seguimiento promedio es de 43 meses $(2,5-$ 149). La sobrevida global es de $95 \%$. La sobrevida específica por cáncer es de $100 \%$ y la sobrevida libre de recurrencia de 97\% (Tabla 1).

\section{Duodeno}

Un paciente de género masculino de 75 años fue sometido en noviembre de 2018, a una DES por un adenoma con displasia de alto grado de la primera porción del duodeno. Se logró una resección en bloque, sin complicaciones y curativa de acuerdo al análisis histológico final.

El paciente se mantiene en controles endoscópicos anuales, sin registrar recurrencia.

\section{Colon y recto}

Diecinueve DES fueron realizadas en 18 pacientes por neoplasias colorrectales entre junio de 2017 y marzo de 2020. El 32\% de las lesiones se ubicaba en el recto, $32 \%$ en el colon izquierdo, $26 \%$ en el colon derecho y $10 \%$ en el transverso.

La histología postoperatoria identificó 6 adenocarcinomas (32\%), 8 adenomas con DAG (42\%) y 5 adenomas con DBG (26\%). 
Tabla 1. Tabla resumen

\begin{tabular}{|c|c|c|c|c|}
\hline Ubicación & $\mathbf{n}$ & Resección & Curación & Sobrevida \\
\hline Esófago & 10 & $\begin{array}{l}\text { En bloc } 100 \% \\
\text { R0 } 100 \%\end{array}$ & $90 \%$ & $\begin{array}{c}\text { Global } 90 \% \\
\text { Específica por cáncer 100\% }\end{array}$ \\
\hline Estómago & 102 & $\begin{array}{l}\text { En bloc } 98 \% \\
\text { R0 } 93,2 \%\end{array}$ & $85 \%$ & $\begin{array}{c}\text { Global 95\% } \\
\text { Específica por cáncer 100\% }\end{array}$ \\
\hline Duodeno & 1 & $\begin{array}{l}\text { En bloc } 100 \% \\
\text { R0 } 100 \%\end{array}$ & $100 \%$ & $\begin{array}{c}\text { Global } 100 \% \\
\text { Específica por cáncer 100\% }\end{array}$ \\
\hline Colon y Recto & 19 & $\begin{array}{l}\text { En bloc } 100 \% \\
\text { R0 } 100 \%\end{array}$ & $100 \%$ & $\begin{array}{c}\text { Global } 100 \% \\
\text { Específica por cáncer 100\% }\end{array}$ \\
\hline Total & 132 & $\begin{array}{l}\text { En bloc } 98,4 \% \\
\text { R0 } 95 \%\end{array}$ & $88 \%$ & $\begin{array}{c}\text { Global } 95 \% \\
\text { Específica por cáncer 100\% }\end{array}$ \\
\hline
\end{tabular}

La resección en bloque fue lograda en todos los casos. Un paciente presentó un síndrome post-polipectomía y fue sometido a una laparoscopía exploradora sin evidenciar perforación, con evolución postoperatoria favorable. días.

La mediana de estadía hospitalaria fue de dos

Todas las resecciones fueron catalogadas como R0 y en todos los pacientes se consideró la DES como curativa de acuerdo a la anatomía patológica.

No se han diagnosticado recurrencias. El promedio de seguimiento fue de 13 meses (3-35). La sobrevida global y específica por cáncer fue de 100\% (Tabla 1).

\section{Discusión}

Los cánceres de tubo digestivo constituyen un importante problema de salud a nivel global. Dan cuenta de cerca de 20\% de todos los nuevos cánceres diagnosticados y de cerca de un cuarto de la mortalidad anual por cáncer en nuestro país $^{1}$. A nivel mundial, estrategias de tamizaje son implementadas con el objetivo de disminuir la mortalidad asociada. A manera de ejemplo, se recomienda realizar una colonoscopia a partir de los 50 años, con el propósito de diagnosticar y tratar lesiones adenomatosas que en el tiempo pudieran derivar en un adenocarcinoma ${ }^{5}$.

La mayor parte de las NITD corresponden a lesiones polipoideas pequeñas que son removidas mediante una $\mathrm{EMR}^{11}$, con bajo riesgo, en las dis- tintas unidades de endoscopía de nuestro país. A medida que el tamaño de las lesiones es mayor, la EMR se vuelve más compleja, frecuentemente resulta en resecciones en fragmentos y aunque macroscópicamente parezca completa, se asocia a mayores tasas de recurrencia ${ }^{11}$. Esto debido a que focos microscópicos inadvertidos pueden permanecer en los bordes laterales o profundos del lecho de resección.

Otro problema asociado a una resección en fragmentos, es que en paralelo al aumento de tamaño de las lesiones, aumenta el riesgo de que contengan un adenocarcinoma ${ }^{21}$. De no ser resecado en una sola pieza, su análisis histopatológico se dificulta significativamente y no permitirá de forma categórica distinguir aquellos pacientes en los cuales la resección endoscópica es el tratamiento definitivo, de quienes deberán ser derivados a cirugía ${ }^{11}$. Esto puede resultar en que pacientes que habiendo sido "curados" se sometan a una intervención innecesaria o que en otros, la EMR sea considerada el tratamiento definitivo sin serlo, comprometiendo el resultado oncológico a largo plazo.

Tradicionalmente la cirugía resectiva o radical ha sido el estándar terapéutico para lograr el mejor resultado oncológico ante el diagnóstico de un cáncer digestivo localizado ${ }^{22,23}$. Es así como la esofagectomía, gastrectomía, pancreato-duodenectomía y colectomías son realizadas para el tratamiento de estas neoplasias. Sin embargo, su morbilidad y mortalidad son elevadas ${ }^{23-29}$ y pueden incluso superar el riesgo oncológico atribuible a lesiones incipientes. 
En un esfuerzo por disminuir el riesgo de recurrencia y evitar los resultados oncológicos adversos de una resección endoscópica incompleta o no evaluable, es que surge la DES. Esta técnica mantiene el paradigma de la cirugía radical, porque logra la resección en bloque de una lesión y por ende, tendría potencial curativo ${ }^{12}$. Su morbilidad baja y nula mortalidad ${ }^{30}$, sumado a la posibilidad de preservar el órgano, reduciendo el impacto en la calidad de vida ${ }^{31}$, la hacen una técnica con potenciales ventajas sobre la cirugía resectiva. En particular en nuestra serie $88 \%$ de los pacientes cumplieron con estándares de curación y la mediana de estadía hospitalaria fue de dos días. Lo anterior se traduce en que 9 de cada 10 pacientes evitaron una cirugía (Tabla 1).

En Chile, la primera DES publicada fue realizada el año 2008 en nuestro centro ${ }^{17}$. En aquellos años la disponibilidad de equipamiento, especialmente en el área de endoscopía, era escasa. No se contaba con insuflación de $\mathrm{CO}_{2}$ ni equipos de alta definición, lo que aumentaba las probabilidades de complicación y no permitía manejarlas con estos recursos. En la actualidad, ha aumentado la disponibilidad de la tecnología e instrumental necesario para realizar procedimientos endoscópicos en condiciones óptimas. En parte esto nos ha permitido reducir las complicaciones de la DES a estándares comparables a las series japonesas ${ }^{30}$.

Probablemente la duda más importante a resolver, derivada de la implementación de esta técnica, es el resultado oncológico en el largo plazo comparado con la cirugía. Diversas series internacionales han demostrado sobrevida a 5 años y sobrevida específica por cáncer equivalentes entre ambas técnicas y solo una ventaja en términos de sobrevida libre de recurrencia para la cirugía ${ }^{7,8,32,33}$. Si bien nuestra serie presenta aún un promedio de seguimiento de 38 meses los resultados obtenidos son comparables a dichas series, destacando 95\% de sobrevida global, 100\% de sobrevida específica por cáncer y sobrevida libre de recidiva de $98 \%$.

La presente experiencia corresponde según nuestro conocimiento a la mayor serie reportada en Latinoamérica de DES para el tratamiento de NITD. Nuestros resultados avalan la factibilidad de ser implementada en el sistema de salud chileno así como los buenos resultados oncológicos en el largo plazo, comparables a las grandes series asiáticas publicadas hasta hoy.
Agradecimientos: Los autores agradecen la valiosa colaboración del Dr. Marco Arrese Jiménez, en la revisión de este manuscrito.

\section{Referencias}

1. Bray F, Ferlay J, Soerjomataram I, Siegel R, Torre L, Jemal A. Global cancer statistics 2018: GLOBOCAN estimates of incidence and mortality worldwide for 36 cancers in 185 countries. CA Cancer J Clin. 2018; 68 (6): 394-424.

2. Ajani JA, In H, Sano T, Gaspar L, Erasmus J, Tang L, et al. Stomach. In: Amin MB. (Ed). AJCC Cancer Staging Manual. 8th Ed. Chicago: AJCC; 2017. p. 203.

3. Katai H, Ishikawa, Akazaxa K, Miyashiro I, Tsujitani $\mathrm{S}$, Ono $\mathrm{H}$, et al. Five-year survival analysis of surgically resected gastric cancer cases in Japan: a retrospective analysis of more than 100,000 patients from the nationwide registry of the Japanese Gastric Cancer Association (2001-2007). Gastric Cancer. 2018; 21 (1): 144-54.

4. Njei B, McCarty TR, Birk JW. Trends in esophageal cancer survival in United States adults from 1973 to 2009: A SEER database analysis. J Gastroenterol Hepatol. 2016; 31 (6): 1141-6.

5. Rex D, Boland R, Dominitz J, Giardiello F, Johnson D, Kaltenbach T, et al. Colorectal cancer screening: Recommendations for physicians and patients from the U.S. Multi-Society Task Force on Colorectal Cancer. Am J Gastroenterol. 2017; 112 (7): 1016-30.

6. Hatta W, Gotoda T, Koike T, Masamune A. History and future perspectives in Japanese guidelines for endoscopic resection of early gastric cancer. Dig Endosc. 2020; 32: $180-90$.

7. Abdelfatah MM, Barakat M, Ahmad D, Ibrahim M, Ahmed Y, Kurdi Y, et al. Long-term outcomes of endoscopic submucosal dissection versus surgery in early gastric cancer: a systematic review and meta-analysis. Eur J Gastroenterol Hepatol. 2019; 31 (4): 418-24.

8. Jeon HK, Kim GH, Lee BE, Park DY, Song GA, Kim DH, et al. Long-term outcome of endoscopic submucosal dissection is comparable to that of surgery for early gastric cancer: a propensity-matched analysis. Gastric Cancer. 2018; 21 (1): 133-43.

9. Guo HM, Zhang XQ, Chen M, Huang SL, Zou XP. Endoscopic submucosal dissection vs endoscopic mucosal resection for superficial esophageal cancer. World J Gastroenterol. 2014; 20 (18): 5540-7.

10. Park HC, Kim DH, Gong EJ, Na EH, Ahn JY, Lee JH, et al. Ten-year experience of esophageal endoscopic sub- 
mucosal dissection of superficial esophageal neoplasms in a single center. Korean J Intern Med. 2016; 31 (6): 1064-72.

11. Dumoulin FL, Hildenbrand R. Endoscopic resection techniques for colorectal neoplasia: Current developments. World J Gastroenterol. 2019; 25 (3): 300-7.

12. Gotoda T, Yamamoto H, Soetikno RM. Endoscopic submucosal dissection of early gastric cancer. J Gastroenterol. 2006; 41 (10): 929-42.

13. Gotoda T, Yanagisawa A, Sasako M, Ono H, Nakanishi $\mathrm{Y}$, Shimoda $\mathrm{T}$, et al. Incidence of lymph node metastasis from early gastric cancer: estimation with a large number of cases at two large centers. Gastric Cancer. 2000; 3 (4): 219-25.

14. Ishihara R, Arima M, Iizuka T. Endoscopic submucosal dissection/endoscopic mucosal resection guidelines for esophageal cancer. Digestive Endoscopy. 2020; 32: 45293.

15. Tanaka S, Kashida H, Saito Y, Yahagi N, Yamano H, Saito S, et al. JGES guidelines for colorectal endoscopic submucosal dissection/endoscopic mucosal resection. Dig Endosc. 2015; 27 (4): 417-34.

16. Chirinos JA, Vargas G, Alcántara C, Zapata J. Endoscopic submucosal dissection as treatment for early gastric cancer: Experience at two centers in Lima, Peru. Disección submucosa endoscópica como tratamiento de cáncer gástrico temprano: experiencia en 2 centros de Lima, Perú. Rev Gastroenterol Mex. 2018; 83 (4): 393-9.

17. Galindo J, Rodríguez J, Norero E, Aguayo G, Pruyas M, Nilsen E, et al. Disección submucosa endoscópica en cáncer gástrico incipiente. Rev Chil Cir. 2015; 67 (6): 590-8.

18. Donoso A, Sharp A, Parra-Blanco A, Roa J, Bächler J, Crovari F, et al. Disección submucosa endoscópica en cáncer gástrico incipiente: experiencia inicial en el Hospital Clínico de la Pontificia Universidad Católica de Chile. Rev Med Chile 2015; 143 (10): 1277-85.

19. Mejía R. Desarrollo de la técnica de disección submucosa endoscópica en cáncer gástrico incipiente. Primeros cincuenta casos de un cirujano. Rev Cirugía 2020; 72 (5). [Epub ahead of print].

20. Chaves DM, Moura EG, Milhomem D, Arantes V, Yamazaki K, Maluf F, et al. Initial experience of endoscopic submucosal dissection in Brazil to treat early gastric and esophagheal cancer: a multi-institutional analysis. Arq Gastroenterol. 2013; 50 (2): 148-52.

21. Nusko G, Mansmann U, Altendorf-Hofmann A, Groitl H, Wittekind C, Hahn EG. Risk of invasive carcinoma in colorectal adenomas assessed by size and site. Int J Colorectal Dis. 1997; 12 (5): 267-71.

22. Japanese Gastric Cancer Association. Japanese gastric cancer treatment guidelines 2014 (ver. 4) Gastric Cancer. 2017; 20 (1): 1-19.

23. Low DE, Kuppusamy MK, Alderson D, Cecconello I, Chang A, Darling G, et al. Benchmarking Complications Associated with Esophagectomy. Ann Surg. 2019; 269 (2): 291-8.

24. Norero E, Ceroni M, Martínez C, Mejía R, Muñoz R, Godoy C, et al. Complicaciones postoperatorias en esofagectomía por cáncer. Evaluación de 215 casos según definiciones del grupo de consenso internacional. Rev Cirugía. 2020; 72 (5). [Epub ahead of print]

25. Norero E, Ceroni M, Ramírez A, Mejía R, Martínez C, Muñoz R, et al. Experiencia inicial con esofagectomia mínimamente invasiva transtorácica en posición semiprono por cáncer esofágico. Rev Chil Cir. 2018; 70 (1): 27-34.

26. Kim H, Hyung W, Cho G, Kim M, Han S, Kim W, et al. Morbidity and mortality of laparoscopic gastrectomy versus open gastrectomy for gastric cancer: An interim report-A phase III multicenter, prospective, randomized trial (KLASS Trial). Ann Surg. 2010; 251 (3): 417-20.

27. Songun I, Putter H, Kranenbarg EM, Sasako M, van de Velde CJ. Surgical treatment of gastric cancer: 15-year follow-up results of the randomised nationwide Dutch D1D2 trial. Lancet Oncol. 2010; 11 (5): 439-49.

28. Longo WE, Virgo KS, Johnson FE, Oprian C, Vernava A, Wade T, et al. Risk factors for morbidity and mortality after colectomy for colon cancer. Dis Colon Rectum. 2000; 43 (1): 83-91.

29. Narayanan S, Martin AN, Turrentine FE, Bauer TW, Adams RB, Zaydfudim VM. Mortality after pancreaticoduodenectomy: assessing early and late causes of patient death. J Surg Res. 2018; 231: 304-8.

30. Oda I, Gotoda T, Hamanaka H, Eguchi T, Saito Y, Matsuda T, et al. Endoscopic submucosal dissection for early gastric cancer: technical feasibility, operation time and complications from a large consecutive cases. Dig Endosc. 2005; 17 (1): 54-8.

31. Kim SG, Ji SM, Lee NR, Park SH, You JH, Choi IJ, et al. Quality of Life after Endoscopic Submucosal Dissection for Early Gastric Cancer: A Prospective Multicenter Cohort Study. Gut Liver. 2016; 11 (1): 87-92.

32. Abe S, Ishihara R, Takahashi H, Ono H, Fujisaki J, Matsui A, et al. Long-term outcomes of endoscopic resection and metachronous cancer after endoscopic resection for adenocarcinoma of the esophagogastric junction in Japan. Gastrointest Endosc. 2019; 89: 1120-8.

33. Morino M, Risio M, Bach S, Beets-Tan R, Bujko K, Panis $\mathrm{Y}$, et al. Early rectal cancer: the European Association for Endoscopic Surgery (EAES) clinical consensus conference. Surg Endosc. 2015; 29: 755-73. 